\title{
The Cox-Maze IV procedure for atrial fibrillation is equally efficacious in patients with rheumatic and degenerative mitral valve disease
}

\author{
Jonathan E. Labin, BS, Nowrin Haque, BA, Laurie A. Sinn, RN, BSN, Richard B. Schuessler, PhD,
} Marc R. Moon, MD, Hersh S. Maniar, MD, Spencer J. Melby, MD, and Ralph J. Damiano, Jr, MD

\section{ABSTRACT}

Objective: To determine whether the etiology of mitral valve disease (MVD), due to either rheumatic or degenerative pathology, influences long-term outcomes after the Cox-Maze IV procedure (CMPIV).

Methods: Between February 2001 and July 2015, 245 patients received a CMIV and concomitant mitral valve procedure. Patients were separated into 2 cohorts based on their etiology of MVD, degenerative $(\mathrm{n}=153)$ and rheumatic $(n=92)$. Patients were followed prospectively (mean follow-up: $41 \pm 37$ months) for recurrent atrial tachyarrhythmias (ATAs). Perioperative variables and longterm freedom from ATAs on and off antiarrhythmic drugs (AADs) were analyzed retrospectively.

Results: The 2 groups differed in that patients with rheumatic MVD were younger, more likely female, had a larger preoperative left atrial diameter, a longer duration of atrial fibrillation (AF), a greater percentage of longstanding persistent AF, and worse New York Heart Association functional class $(P \leq .001)$. Although there was no difference in operative mortality or overall major complications between the groups, the median length of stay in the intensive care unit was longer in the rheumatic cohort. Freedom from recurrent ATAs through 5 years was similar between the 2 cohorts. Predictors of recurrence included failure to use a box-lesion $(P=.012)$, the duration of preoperative AF $(P=.001)$, and early occurrence of ATAs $(P=.015)$.

Conclusions: The long-term efficacy of the CMPIV in restoring sinus rhythm was similar in patients with either rheumatic or degenerative mitral valve disease. Despite representing a sicker patient population with a longer duration of preoperative AF, patients with rheumatic MVD equally benefit from the CMPIV. (J Thorac Cardiovasc Surg 2017;154:835-44)

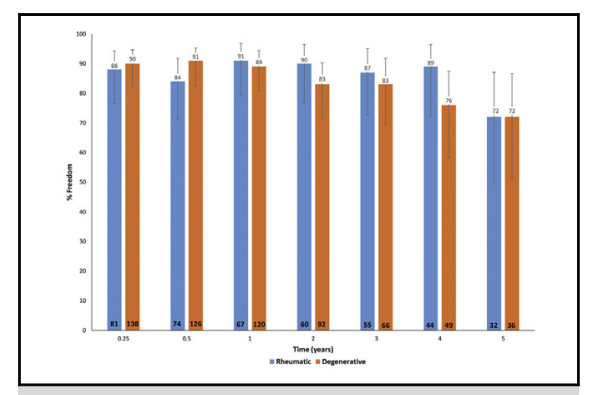

Five-year freedom from atrial tachyarrhythmias was similar regardless of the etiology of mitral valve disease.

\section{Central Message}

The long-term efficacy of the Cox-Maze IV procedure in restoring sinus rhythm is independent of the underlying etiology of mitral valve disease.

\section{Perspective}

This study demonstrates that the long-term efficacy of the Cox-Maze IV procedure in restoring sinus rhythm is similar in patients with either rheumatic or degenerative mitral valve disease. It addresses the disputed evidence regarding the efficacy of the Cox-Maze IV procedure and should encourage the wider application of surgical ablation in all patients with $\mathrm{AF}$ and mitra valve disease, irrespective of their left atrial size or other comorbidities.

See Editorial Commentary page 845

\footnotetext{
From the Division of Cardiothoracic Surgery, Department of Surgery, Washington University School of Medicine, Barnes-Jewish Hospital, St Louis, Mo.

This study was funded by National Institutes of Health grants T35 NHLBI, 5R01 HL32257, and T32 HR07275.

Read at the 96th Annual Meeting of The American Association for Thoracic Surgery Annual Meeting, Baltimore, Maryland, May 14-18, 2016.

Received for publication May 15, 2016; revisions received Jan 23, 2017; accepted for publication March 9, 2017; available ahead of print June 2, 2017.

Address for reprints: Ralph J. Damiano, Jr, MD, Division of Cardiothoracic Surgery, Washington University School of Medicine, Barnes-Jewish Hospital, 660 S. Euclid Ave, Campus Box 8234, St. Louis, MO 63110 (E-mail: Damianr@wustl.edu). $0022-5223 / \$ 36.00$

Copyright $(C) 2017$ Published by Elsevier Inc. on behalf of The American Association for Thoracic Surgery

http://dx.doi.org/10.1016/j.jtcvs.2017.03.152
}

With an estimated worldwide prevalence of 33.5 million, atrial fibrillation (AF) is the most common cardiac arrhythmia and a significant risk factor for thromboembolic strokes. ${ }^{1}$ Because of the poor efficacy of

Scanning this QR code will take you to a supplemental video. To view the AATS 2016 Webcast, see the URL next to the Webcast thumbnail.

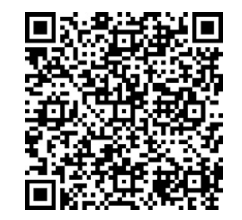




$$
\begin{aligned}
& \text { Abbreviations and Acronyms } \\
& \text { AAD }=\text { antiarrhythmic drug } \\
& \text { AF }=\text { atrial fibrillation } \\
& \text { ATA }=\text { atrial tachyarrhythmia } \\
& \text { CMPIV }=\text { Cox-Maze IV procedure } \\
& \text { ICU }=\text { intensive care unit } \\
& \text { LA }=\text { left atrial } \\
& \text { MV }=\text { mitral valve } \\
& \text { MVD }=\text { mitral valve disease } \\
& \text { NYHA } \\
& \text { OR New York Heart Association } \\
&
\end{aligned}
$$

pharmacologic therapy, a number of interventional procedures have been introduced to treat AF. The first and most successful procedure was introduced in 1987 by $\mathrm{Dr}$ James Cox and was termed the Cox-Maze procedure. Since its conception, the Cox-Maze procedure has been refined over several iterations, the latest of which, the Cox-Maze IV procedure (CMPIV), uses bipolar radiofrequency ablation and cryoablation. ${ }^{2}$ With the advent of the CMPIV and its decreased operative times, an increasing percentage of patients currently receiving the CMPIV are undergoing concomitant mitral valve (MV) surgery, an approach that does not affect morbidity and mortality. ${ }^{3-6}$

AF commonly is associated with mitral valve disease (MVD) and is present in up to one third of patients referred for mitral surgery. ${ }^{1,7}$ It is the most common indication for surgical ablation of $\mathrm{AF}$, with more than one half of all AF operations being performed in patients undergoing MV procedures. ${ }^{6}$ Although the CMPIV and concomitant MV procedure has been established as a highly efficacious treatment for AF associated with nonrheumatic MVD, the relative success of the CMPIV in patients with rheumatic MVD remains disputed. $^{3,8,9}$ Indeed, in patients with rheumatic MVD, some investigators have reported the CMPIV as less effective in the treatment of drug-refractory $\mathrm{AF}^{7,9}$ Considering that rheumatic heart disease is the most commonly acquired heart disease in developing countries, affecting nearly 20 million people worldwide, it is critical to characterize the efficacy of the CMPIV in this population. ${ }^{10}$ The purpose of this study was to determine whether the underlying etiology of MVD, as the result of either rheumatic or degenerative processes, affects the long-term outcomes of the CMPIV.

\section{METHODS}

This study was approved by the Washington University School of Medicine Institutional Review Board. Informed consent and permission for release of information was obtained from each participant. All data were entered prospectively into a longitudinal database maintained at our institution.

\section{Patients}

From February 2001 to July 2015, 245 patients with AF underwent a CMPIV and concomitant MV procedure. The patients were divided into 2 cohorts based on the underlying etiology of their MVD, rheumatic (92) and degenerative (153). All patients with nonrheumatic/nondegenerative MVD were excluded from this study. This exclusion criteria encompassed patients with MVD secondary to endocarditis, annular dilatation, calcification, congenital heart disease, ischemic heart disease, or prosthetic valve dysfunction. Patients were screened for inclusion with the Washington University School of Medicine institutional AF database that prospectively records all patients, and their associated demographics and peri- and postoperative events, who underwent a Cox-Maze procedure at BarnesJewish Hospital. Of the 323 patients initially screened for inclusion, 245 patients met the parameters for the study.

Rheumatic MVD was determined by history of acute rheumatic fever or scarlet fever, gross valve morphology, and echocardiographic findings. Echocardiographic and intraoperative findings of commissural and/or chordal fusion, leaflet thickening and retraction, and leaflet doming and mitral stenosis were considered rheumatic MVD. The lesion set was similar among all patients except for the addition of a superior left atrial (LA) lesion in 2005, which connected the right and left superior pulmonary veins; an addition classified as a "box-lesion" set. ${ }^{11}$ The LA appendage was managed in all patients. Most were either excised or oversewn and, rarely, a clip was used to facilitate closure.

Patients were discharged on class I or III antiarrhythmic drugs (AADs) and warfarin, unless contraindicated. AADs were discontinued 2 months postoperatively if patients maintained normal sinus rhythm. Patients were maintained or restarted on AADs if they demonstrated recurrence of AF. The specific drug and duration of treatment of AADs was adjudicated by the patient's cardiologist and cardiothoracic surgeon and primarily driven by evidence of normal sinus rhythm and meaningful atrial contraction. Anticoagulation was discontinued between 3 and 6 months in patients with no evidence of recurrent atrial tachyarrhythmias (ATAs) on prolonged monitoring and adequate atrial emptying as assessed by a transthoracic echocardiogram performed on all patients postoperatively. $\beta$-Blockers and calcium channel blockers were not considered as AADs. The authors of this study had primary oversight of postoperative management and monitoring. Indeed, one surgeon performed the majority of procedures and guided follow-up and postoperative treatment for most of the patients in this study.

\section{Study Design}

The patients' clinical profiles and postoperative outcomes were recorded prospectively in our institutional computerized database. Demographic and perioperative variables $(\mathrm{n}=27)$ including complications were compared between the 2 groups. Follow-up was conducted and obtained at 3 months, 6 months, and then annually. Patients were followed prospectively and were monitored for recurrent ATAs via Holter monitoring, pacemaker interrogation, interrogation of implantable cardiac loop recorders, or electrocardiograms. Late recurrence was defined as per the Heart Rhythm Society guidelines: any episode of AF, atrial flutter, or atrial tachycardia that lasted longer than 30 seconds and occurred outside of the immediate 3-month postoperative period. ${ }^{12}$ Patients who experienced any episode of AF within 3 months of surgery were considered early ATAs as defined by the Heart Rhythm Society. Late recurrence is defined as any episode of $\mathrm{AF}$ after the 3-month blanking period. Any patient who required an interventional electrophysiological procedure was classified as a permanent failure.

\section{Statistical Analysis}

Continuous variables are expressed as mean \pm standard deviation or as a median with a range. Categorical variables are expressed as frequencies and percentages with outcomes compared with the $\chi^{2}$ or the Fisher exact test. Continuous outcomes were compared with the $t$ test on normally 
TABLE 1. Type of concomitant procedures

\begin{tabular}{lcc}
\hline Concomitant operation & $\begin{array}{c}\text { Rheumatic } \\
(\mathbf{n = 9 2 )}\end{array}$ & $\begin{array}{c}\text { Degenerative } \\
(\mathbf{n = 1 5 3 )}\end{array}$ \\
\hline MV repair & $5(5 \%)$ & $92(60 \%)$ \\
MV repair + TVR & $2(2 \%)$ & $12(8 \%)$ \\
MV repair + AVR & 0 & $3(2 \%)$ \\
MV repair + CABG & $1(1 \%)$ & $18(12 \%)$ \\
MV repair + other & 0 & $1(1 \%)$ \\
MVR & $55(60 \%)$ & $16(10 \%)$ \\
MVR + TVR & $11(12 \%)$ & $2(1 \%)$ \\
MVR + AVR & $8(9 \%)$ & $1(1 \%)$ \\
MVR + CABG & $4(4 \%)$ & $4(3 \%)$ \\
MVR + other & $6(7 \%)$ & $4(3 \%)$ \\
\hline
\end{tabular}

$M V$, Mitral valve; $T V R$, tricuspid valve replacement; $A V R$, aortic valve replacement; $C A B G$, coronary artery bypass graft; $M V R$, mitral valve replacement.

distributed continuous variables. The Mann-Whitney $U$ nonparametric test was used for skewed distributions.

Thirty-eight pre- and perioperative variables were evaluated by univariate analysis to identify predictors of AF recurrence at 1 year. Variables used in univariate analyses included the MVD etiology in addition to those listed in Tables 1 and 2, with the exception of mortality and intensive care unit (ICU) length of stay. Significant covariates on univariate analysis $(P \leq .10)$ or covariates deemed clinically relevant based on previous experience were entered into a stepwise multivariate binary logistic regression analysis to determine the independent predictors of recurrence at 1 year. ${ }^{5}$ All data analyses were performed with SYSTAT 13 software (Systat Software Inc, Chicago, Ill).

\section{RESULTS}

Of the 153 patients who received a CMIV and concomitant MV procedure, 92 (38\%) had rheumatic MVD and 153 $(62 \%)$ had degenerative MVD. MV operations included patients receiving an MV repair (134 patients, 55\%) or MV replacement (111 patients, 45\%). However, the majority of patients suffering from rheumatic MVD had an MV replacement $(91 \%)$, whereas the majority of patients with degenerative MVD received MV repair procedures $(82 \%)$ (Table 1).
The average follow-up time within the rheumatic cohort was $48 \pm 39$ months compared with $36 \pm 34$ months in the group with degenerative MVD. Overall, the mean follow-up time for all patients was $41 \pm 37$ months (median follow-up of 36 months). Among both cohorts, $76 \%$ of patients received prolonged follow-up via Holter monitoring, pacemaker interrogation, and/or interrogation of implantable loop recorders. Of those patients with rheumatic MVD, $70 \%$ received prolonged monitoring compared with the $80 \%$ in the degenerative MVD cohort. All but one of the patients who were followed and monitored for recurrent ATAs received the latest iteration of the Cox-Maze Procedure, the CMPIV.

\section{Demographics}

There were significant preoperative differences between the groups in terms of age, sex, body mass index, New York Heart Association (NYHA) classification, type and duration of preoperative AF, and LA diameter (Table 2). Although the degenerative cohort had a smaller LA diameter and a shorter AF duration, the rheumatic MVD group had a significantly greater body mass index $\left(\mathrm{kg} / \mathrm{m}^{2}\right)$, a worse NYHA functional classification, and a greater percentage of longstanding, persistent $\mathrm{AF}$ despite being younger.

The male-to-female ratio was significantly different between the 2 cohorts (rheumatic MVD 1:3.5, degenerative MVD 1.2:1, $P<.0001)$. To further establish a baseline risk profile and assess the risk of thromboembolism, the $\mathrm{CHA}_{2} \mathrm{DS}_{2}$-VASc score was calculated. ${ }^{13}$ There was no significant difference between the 2 groups $(P=.233)$.

\section{Perioperative Outcomes}

The majority of perioperative outcomes were similar between both groups. However, the median ICU length of stay was significantly longer in patients with rheumatic MVD

TABLE 2. Demographic outcomes

\begin{tabular}{lccc}
\hline \multicolumn{1}{c}{ Variable } & Rheumatic MV etiology $(\mathbf{n}=\mathbf{9 2})$ & Degenerative MV etiology $(\mathbf{n}=\mathbf{1 5 3})$ & $\boldsymbol{P}$ value \\
\hline Age, y & $63 \pm 10$ & $68 \pm 11$ & $<.001$ \\
Sex, \% male & 28 & 54 & $<.0001$ \\
BMI, kg/cm & $29.0 \pm 6.56$ & $27.2 \pm 6.49$ & .038 \\
AF duration, mo & $48[0-324]$ & $21[0.3-420]$ & .035 \\
Paroxysmal AF, \% & 38 & 47 & .185 \\
Persistent AF, \% & 10 & 35 & .096 \\
Long-standing, persistent AF, \% & 49 & 58 & .032 \\
NYHA III or IV, \% & 80 & 8 & $<.001$ \\
Failed catheter ablation, \% & 8 & 11 & .806 \\
Preoperative pacemaker, \% & 8 & $5.25 \pm 1.08$ & .506 \\
LA diameter, cm & $5.80 \pm 1.06$ & $4[1-7]$ & 16.3 \\
CHA ${ }_{2}$ DS $_{2}$-Vasc & $4[1-7]$ & .001 \\
Prior thromboembolism, \% & 18.5 & .233 \\
\hline AF
\end{tabular}

AF duration is listed as total number of months; continuous variables are listed as mean \pm standard deviation. $M V$, Mitral valve; $B M I$, body mass index; $A F$, atrial fibrillation; NYHA, New York Heart Association classification; $L A$, left atrial. 
TABLE 3. Perioperative outcomes

\begin{tabular}{|c|c|c|c|}
\hline Variable & Rheumatic MV etiology $(n=92)$ & Degenerative MV etiology $(n=153)$ & $P$ value \\
\hline Box-lesion, $\%$ & $81(88 \%)$ & $140(92 \%)$ & .384 \\
\hline Cardiopulmonary bypass time, $\min$ & $189.61 \pm 41.0$ & $195.21 \pm 46.3$ & .326 \\
\hline Crossclamp time, min & $100.22 \pm 25.3$ & $102.12 \pm 30.3$ & .598 \\
\hline Permanent pacemaker & $13(14 \%)$ & $22(14 \%)$ & 1 \\
\hline Overall major complications & $19(22 \%)$ & $30(19 \%)$ & .547 \\
\hline Pneumonia & $9(10 \%)$ & $16(10 \%)$ & 1 \\
\hline Deep sternal infection & $0(0 \%)$ & $0(0 \%)$ & 1 \\
\hline Intra-aortic balloon pump & $3(3 \%)$ & $6(4 \%)$ & 1 \\
\hline Permanent stroke & $3(3 \%)$ & $3(2 \%)$ & .675 \\
\hline Reoperation for bleeding & $9(10 \%)$ & $16(10 \%)$ & 1 \\
\hline Renal failure requiring dialysis & $9(10 \%)$ & $10(7 \%)$ & .460 \\
\hline Median ICU LOS in days, range & $4[1-78]$ & $3[1-62]$ & .002 \\
\hline 30-d mortality, \% & $4(4.3 \%)$ & $6(3.9 \%)$ & 1 \\
\hline Long-term incidence of thromboembolism & $6(6.7 \%)$ & $9(6.4 \%)$ & .926 \\
\hline
\end{tabular}

Continuous variables are listed as mean \pm standard deviation; complications are listed as total number (\%). $M V$, Mitral valve; $I C U$, intensive care unit; $L O S$, length of stay.

(4 vs 3 days, $P=.002$, Table 3 ). Major complications, as listed in Table 3, were defined as pneumonia, deep sternal infection, need for an intra-aortic balloon pump, permanent stroke, reoperation for bleeding, and renal failure requiring dialysis. There were no differences between the groups in terms of operative or postoperative variables and complications. In addition, there was no difference in operative mortality or the long-term incidence of thromboembolism $(P=1$ and 0.926 , respectively). There was also no difference in postoperative rates of stroke or thromboembolism when we compared those who received an MV replacement with those patients who received an MV repair. Thromboembolism was defined as a systemic embolism occurring outside the 30-day postoperative period that resulted in a cerebrovascular accident, and permanent pacemaker complication was defined as the placement of a permanent pacemaker any time after surgical ablation.

\section{Efficacy}

Freedom from ATAs after a CMIV and concomitant MV procedure in patients with rheumatic MVD was $88 \%, 84 \%$, $91 \%, 90 \%, 87 \%, 89 \%, 72 \%$ at 3,6 months, and 1 through

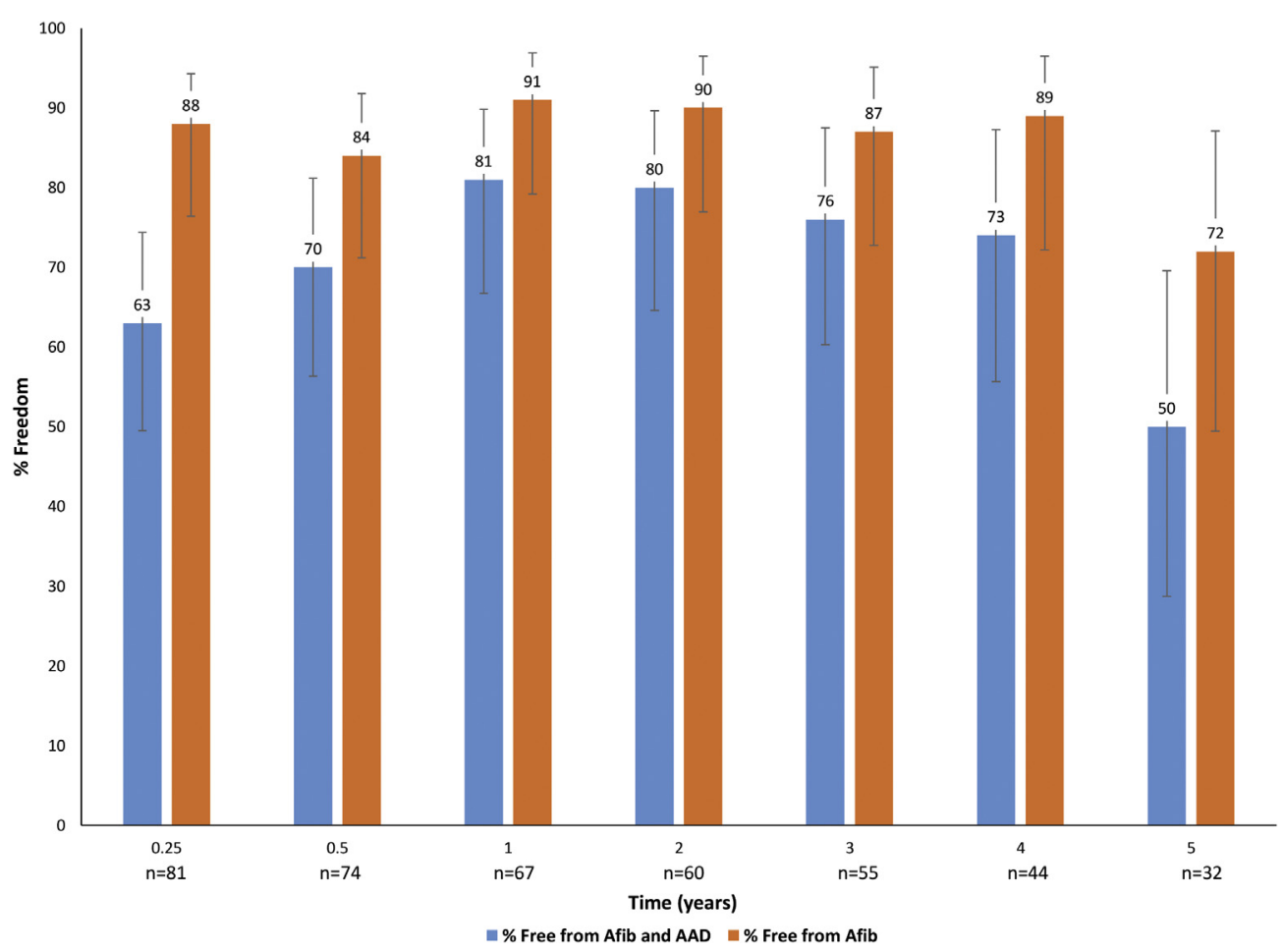

FIGURE 1. Five-year follow-up showing freedom from atrial fibrillation both on and off AADs with $95 \%$ confidence intervals after a concomitant $\mathrm{CMIV}+\mathrm{MV}$ procedure for AF associated with rheumatic MVD. Afib, Atrial fibrillation; $A A D$, antiarrhythmic drug. 


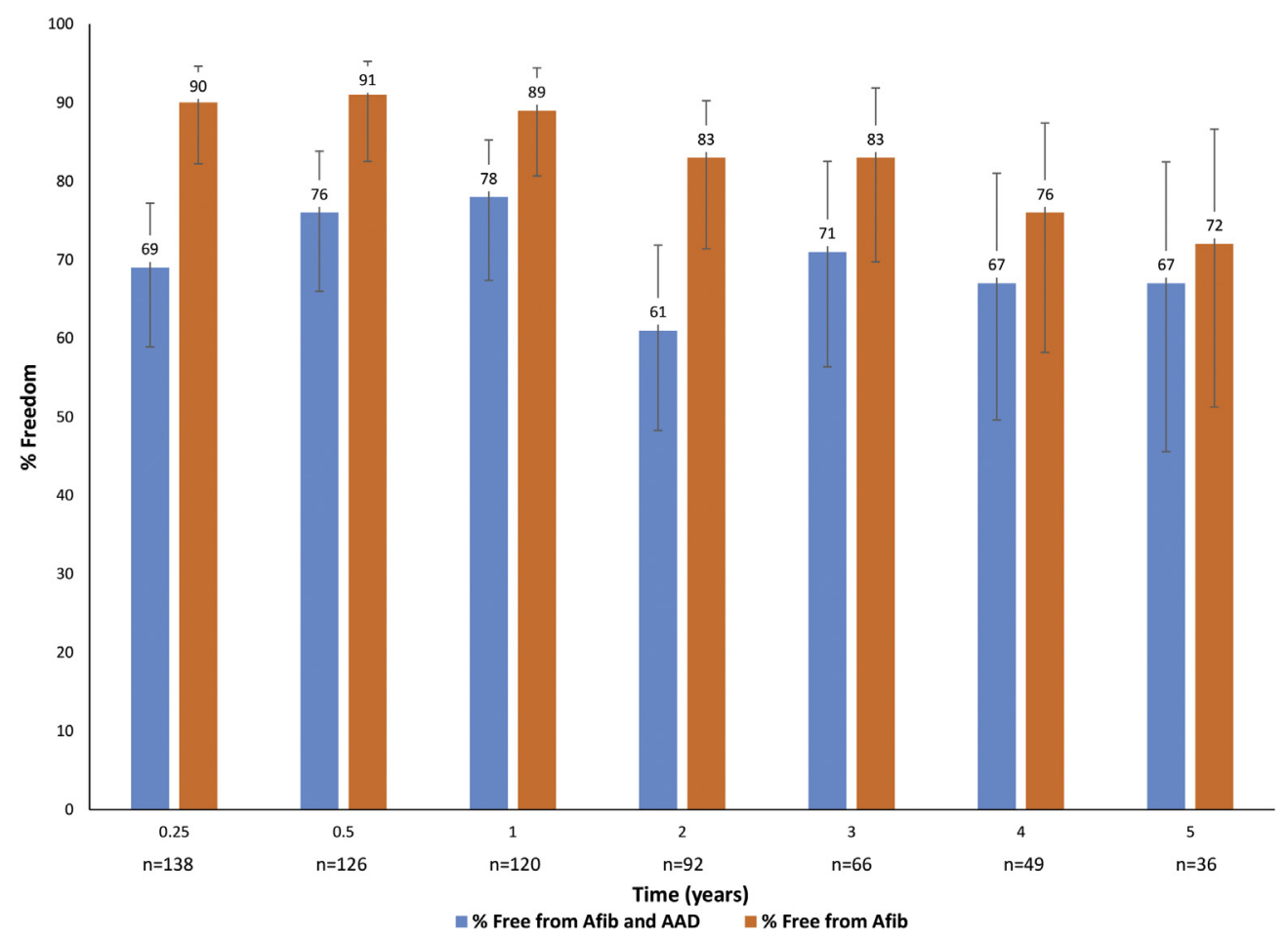

FIGURE 2. Five-year follow-up showing freedom from atrial fibrillation both on and off AADs with $95 \%$ confidence intervals after a concomitant CMIV + MV procedure for AF associated with degenerative MVD. Afib, Atrial fibrillation; AAD, antiarrhythmic drug.

5 years, respectively (Figure 1). In the same group, freedom from both ATAs and AADs was 63\%, 70\%, 81\%, 80\%, $76 \%, 73 \%$, and $50 \%$ at the same time points. When a CMIV and MV procedure was performed in patients with degenerative MVD, the freedom from ATAs was $90 \%$, $91 \%, 89 \%, 83 \%, 83 \%, 76 \%, 72 \%$ at 3,6 months, and 1 through 5 years, respectively (Figure 2). Freedom from ATAs and AADs in patients with degenerative MVD was $69 \%, 76 \%, 78 \%, 61 \%, 71 \%, 67 \%$, and $67 \%$ at the same time points. There was no significant different in freedom from AF on and off AADs between the 2 cohorts through 5 years (Figure 3). Notably, comparison of freedom from $\mathrm{AF}$ and AADs between the 2 groups showed no significant difference at all years except for year $2(P=.020$, Figure 4). The denominator used to determine these percentages is based on the number of patients that were followed-up successfully during the study at each specific time point. This includes all patients on which data were obtained regarding their rhythm status and anticoagulation/ AAD use. Of note, the follow-up available for the combined cohorts at 3 months, 6 months, and 1 through 5 years was 96\% (219/229), 91\% (200/220), 89\% (187/211), 85\% (152/179), 85\% (121/143), 84\% (93/111), and 83\% (68/ $82)$, respectively.

\section{Risk of Recurrence}

The following were univariate predictors of recurrence at 1 year: longstanding, persistent $\mathrm{AF}(P=.003)$, renal failure requiring dialysis $(P=.055)$, preoperative pacemaker $(P=.002)$, box lesion $(P=.001)$, length of time in preoperative $\operatorname{AF}(P=.003)$, early occurrence of ATAs $(P=.002)$, and crossclamp time $(P=.002)$. The etiology of MVD was not a predictor $(P=.620)$. These variables were all included in a stepwise logistic regression model. In the combined group of patients, the multivariate predictors of $\mathrm{AF}$ recurrence at 1 year included failure to use a box-lesion to isolate the posterior left atria (odds ratio [OR], 0.248, $P=.012$ ), the duration of preoperative AF in years (OR, 1.093; $P=.001$ ), and early occurrence of ATAs (OR, 3.192; $P=.015)$. The area under the receiver operating characteristic curve was 0.750 .

\section{DISCUSSION}

This study evaluated whether the underlying etiology of MVD, due to either a rheumatic or degenerative process, affected late outcomes of the CMIV and concomitant MV procedure in treating AF. Although the CMPIV has been shown to be efficacious in the treatment of $\mathrm{AF}$ associated with nonrheumatic MVD, the efficacy of the CMPIV in treating $\mathrm{AF}$ associated with rheumatic MVD has remained controversial. Based on the prevalence of rheumatic heart disease and its common association with $\mathrm{AF}$, it is critical to accurately characterize the efficacy of the CMPIV in treating patients suffering from AF associated with rheumatic MVD.

Although previous series regarding $\mathrm{AF}$ associated with rheumatic MVD predominately have investigated the 


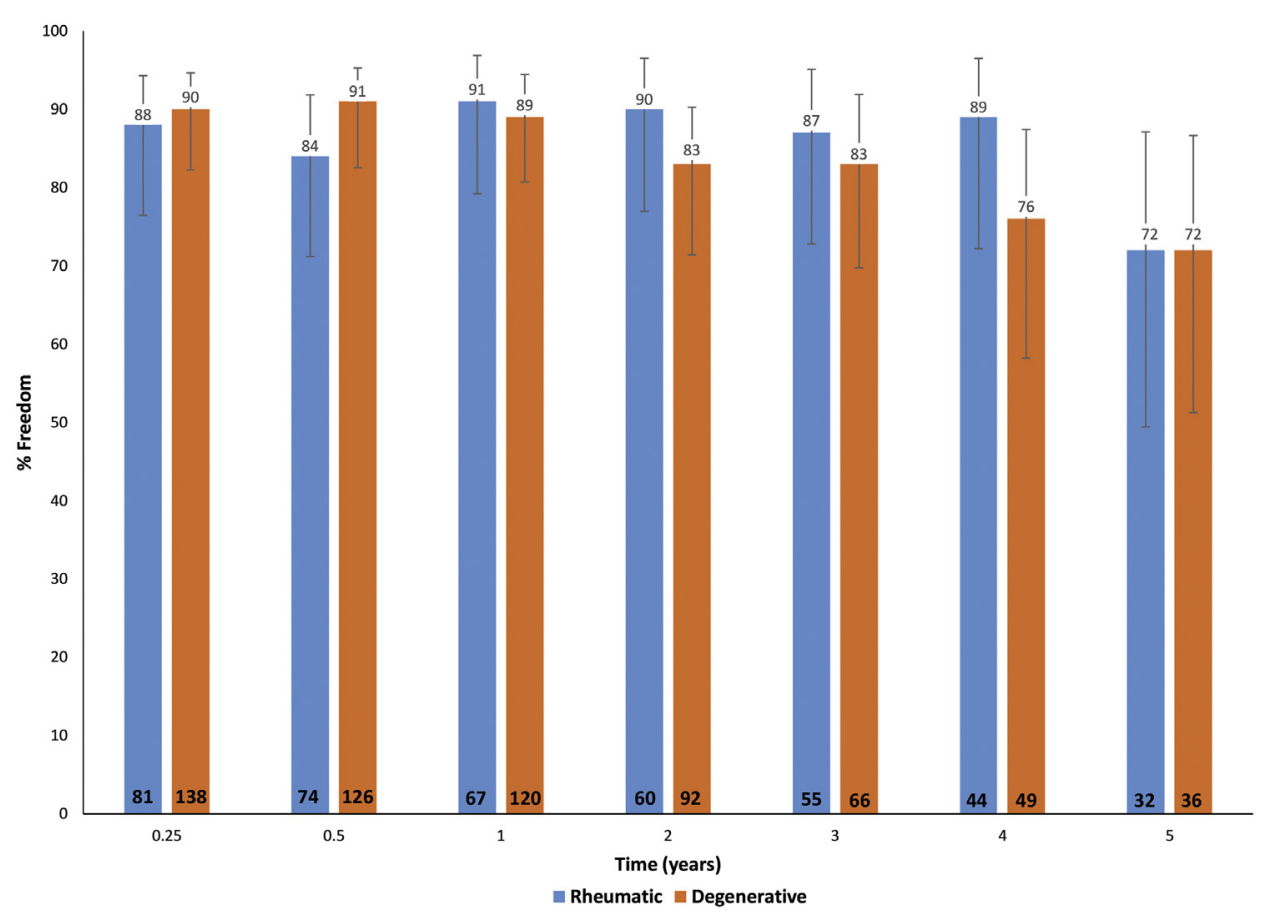

FIGURE 3. Five-year follow-up comparing freedom from atrial fibrillation on and off AADs with $95 \%$ confidence intervals after a concomitant $\mathrm{CMIV}+\mathrm{MV}$ procedure for AF associated with rheumatic versus degenerative MVD. There was no significant difference through 5 years $(P>.102)$.

efficacy of the surgical ablation and MV procedure in a single concomitant cohort, few have directly compared the extent to which the etiology of MVD affects the longterm outcomes of the CMPIV. ${ }^{7-9,14}$ As a result, this study offers a unique opportunity to both identify the importance that etiology of MVD plays in regard to the efficacy of the CMPIV and provides evidence to judge whether previous nonrheumatic CMPIV studies can be used to guide clinical decisions in patients suffering from rheumatic MVD. In addition, this study provides further insight into whether there are unique pathophysiologic mechanisms in maintaining AF associated with rheumatic MVD. Specific histologic changes in the atrial myocardium have been cited as significant contributors to the onset and maintenance of $\mathrm{AF}$ associated with rheumatic MVD. ${ }^{9,13,15}$ Fukada and colleagues ${ }^{14}$ suggested that the fibrosis and intracellular changes observed in the myocardium may contribute to the unsatisfactory results of the Cox-Maze procedure in rheumatic patients. However, our results demonstrate that such rheumatic inflammation and associated pathology may not affect the recurrence of $\mathrm{AF}$ after surgical ablation.

Our results clearly show that the CMIV was similarly efficacious in patients with either rheumatic or degenerative MVD up to 5 years postoperatively. The results from this series of patients who underwent CMIV agree with previous reports from both this institution and other centers. Indeed, the percent freedom from AF recurrence through 5 years was similar to other studies that evaluated the success of the Cox-Maze procedure in treating AF associated with rheumatic and degenerative MVD. ${ }^{4,5,7,8}$

As expected, the 2 groups had distinctly different demographics. Interestingly, other than age and sex, the demographic differences between the 2 cohorts suggest that patients suffering from $\mathrm{AF}$ associated with rheumatic MVD were sicker at baseline and would therefore likely experience a greater rate of AF recurrence after the CMPIV. Patients with rheumatic MVD had a larger preoperative LA diameter, $\mathrm{AF}$ of longer duration, a greater percentage of longstanding, persistent $\mathrm{AF}$, and worse NYHA functional class $(P \leq .001)$. However, despite these differences, the CMIV was similarly efficacious regardless of MVD etiology, an outcome particularly noteworthy considering that many of these comorbidities are risk factors for AF recurrence after a surgical ablation, as demonstrated in several surgical series. ${ }^{16-18}$

Despite these distinct demographics, there was no significant difference in major complication rates. However, patients suffering from AF associated with rheumatic MVD stayed in the ICU, on average, 1 day longer than the patients with degenerative MVD. This may reflect their difference in preoperative health status. Both the $\mathrm{CHA}_{2} \mathrm{DS}_{2}$-VASc score and the long-term incidence of thromboembolism were similar between the rheumatic and degenerative MVD cohorts. 


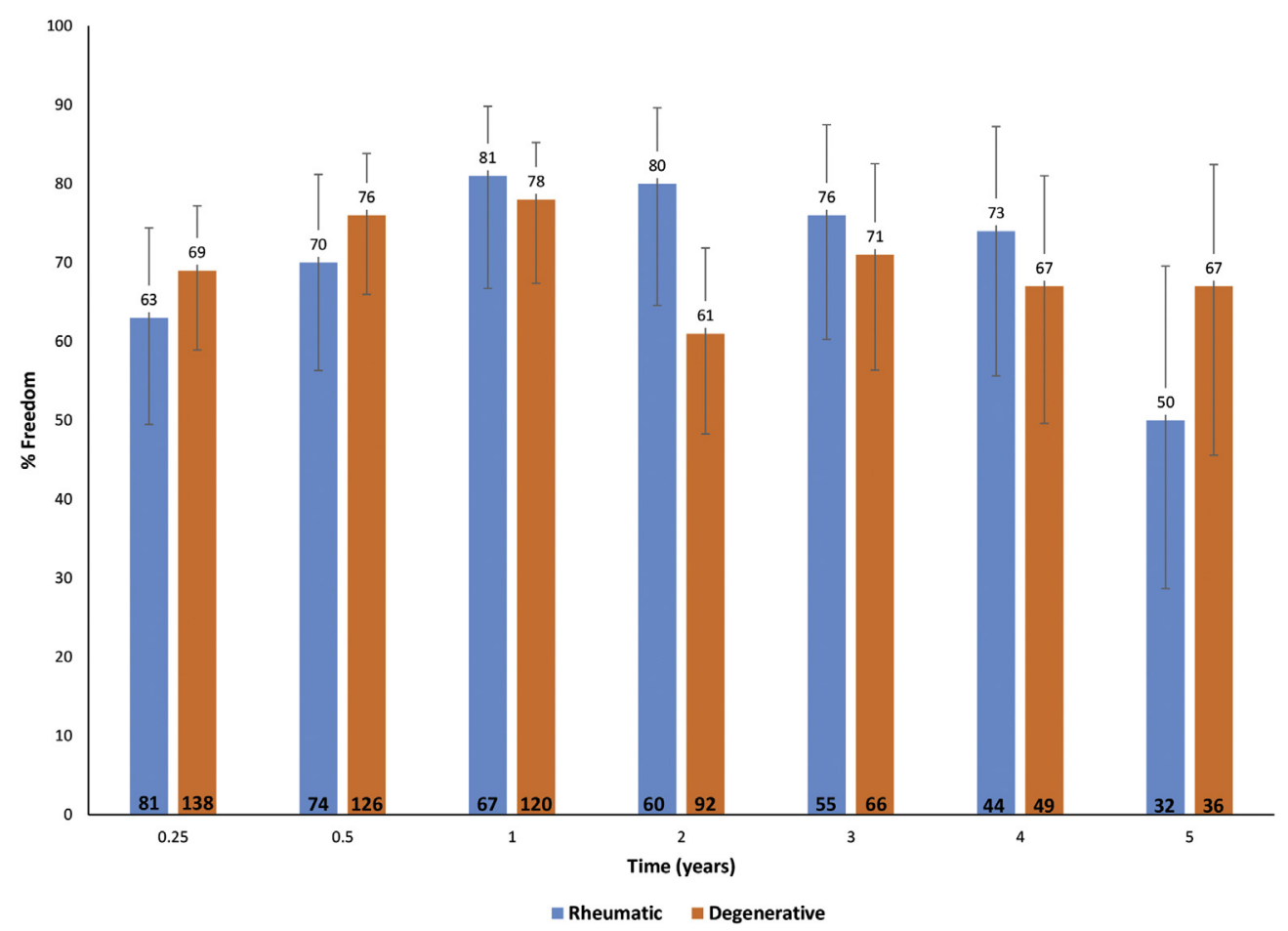

FIGURE 4. Five-year follow-up comparing freedom from atrial fibrillation off antiarrhythmic drugs with $95 \%$ confidence intervals after a concomitant $\mathrm{CMIV}+\mathrm{MV}$ procedure for AF associated with rheumatic versus degenerative MVD. At 2 years, $P=.020$. At all other time points, there was no significant difference $(P>.22)$.

Multivariate analysis identified 3 risk factors for recurrence of ATAs at 1 year postoperatively in this large cohort of more than 250 patients. These include failure to isolate the posterior LA using a box lesion set, the occurrence of early ATAs in the immediate postoperative setting, and the preoperative duration of $\mathrm{AF}$.

As expected, and supported by several previous studies from our group, failure to electrically isolate the posterior LA is strongly associated with failure of the CMPIV, a consistent finding that has led our group to include a box lesion set in all patients undergoing surgical ablation for AF (Video 1). ${ }^{11,19,20}$ Notably, the inclusion of a box lesion to electrically isolate the posterior LA was far more predictive of success and freedom from recurrent ATAs at 1-year follow-up in the rheumatic cohort. Considering that the box lesion set was only introduced in 2005 and the majority of similarly focused studies were conducted before the advent of the CMPIV, this outcome may account for the discrepancy in the medical literature regarding the success of the Maze procedure in treating drug-refractory AF associated with rheumatic MVD. Indeed, perhaps the well-documented pulmonary vein dilatation and chronic inflammatory reaction of the atrial myocardium in rheumatic heart disease mandates a more aggressive and complete ablation, particularly surrounding the arrhythmogenic pulmonary vein sleeves, an approach achieved with the CMPIV.
The early occurrence of postoperative ATAs also was a predictor of late recurrence. This finding, in agreement with previous reports in the literature, suggests that the substrate for early and late ATAs may be similar. ${ }^{21,22}$ Despite these findings, the relevance of early ATAs to clinically significant success with the CMPIV remains unclear. In all patients, $62 \%$ had early ATAs but at 4 and 5 years

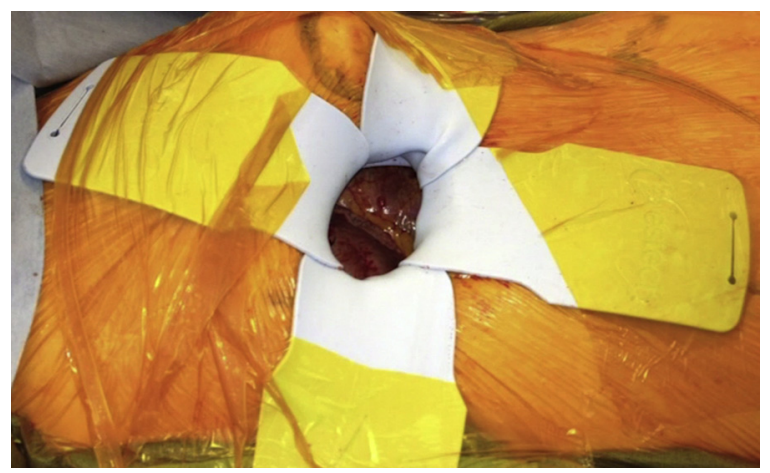

VIDEO 1. Minimally invasive Cox-Maze IV procedure performed by Dr Ralph J. Damiano, Jr. A standard mitral valve repair and annuloplasty follows. Adapted with permission from the AME Publishing Company from Saint LL, Lawrance CP, Leidenfrost JE, Robertson JO, Damiano RJ Jr. How I do it: minimally invasive Cox-Maze IV procedure. Ann Cardiothorac Surg. 2014;3:117-9. Video available at: http://www.jtcvsonline. org/article/S0022-5223(17)30897-8/addons. 
postoperatively, only $24 \%$ and $27 \%$ of those who experienced early ATAs had recurrent AF, respectively. The majority of patients who experienced early ATAs remained free from late recurrence.

The duration of preoperative AF was the most significant predictor of recurrence at 1 year after the Maze procedure. This finding is consistent with previous series and suggests that the chronicity of AF is significant in causing atrial remodeling and fibrosis that lowers the efficacy of the CMPIV. ${ }^{21}$ This would argue for earlier surgical intervention in patients with MVD who develop AF to increase the likelihood of restoring sinus rhythm after surgery.

Unlike previous studies, our data did not demonstrate LA size to be predictive of recurrence of AF after a Cox-Maze procedure. This is an interesting finding and one that may be due to a number of factors. First of all, our center has had a policy of aggressive LA reduction if the LA diameter was $>6 \mathrm{~cm}$. Moreover, with a box isolation of the entire posterior LA, this significantly reduces LA area from an electrical standpoint and this may ameliorate the effect of increased LA size on recurrence. ${ }^{23}$

Although this is one of the largest studies comparing the efficacy of the CMPIV in patients with rheumatic and degenerative MVD, it is not without its limitations. Although both patient cohorts were well-monitored with prolonged follow-up $(70 \%$ rheumatic, $80 \%$ degenerative MVD), some patients did not have 24-hour Holter monitoring and instead were assessed for the presence of ATAs with an electrocardiogram, a method prone to underestimation of the incidence of recurrent AF. Also, although the use of 24-hour continuous cardiac monitoring provides a reasonable measure of $\mathrm{AF}$ recurrence, it fails to address the clinical burden of AF. Indeed, although the current method of 24-hour monitoring does not quantitate burden, future studies using implantable loop recorders could provide this information. Moreover, this study has inherent selection bias considering it is a retrospective review. However, it should be noted that all data were collected prospectively and this was a series of consecutive patients. Finally, although this study demonstrated similar efficacy of the CMPIV through 5 years between both groups, the mechanism of procedural failure was not defined. It remains undetermined whether the recurrence of $\mathrm{AF}$ was due to technical difficulty in completing the lesion set or an inability to eliminate the underlying mechanism of AF in these patients. In certain settings, very small areas of atria can sustain reentry, making it exceedingly difficult for the CMPIV to eliminate $\mathrm{AF}^{23}$

This study emphasizes both the importance of early intervention and the isolation of the entire posterior LA when performing the CMPIV. In addition, and perhaps most importantly, our data indicate that the long-term efficacy of the CMPIV in restoring sinus rhythm was similar in patients with either rheumatic or degenerative MVD up through 5 years. Indeed, despite representing a sicker patient population, with longer duration of preoperative $\mathrm{AF}$ and larger LA diameters, patients with $\mathrm{AF}$ associated with rheumatic MVD may equally benefit from the CMPIV, a finding that should encourage the wider application of surgical ablation in all patients with AF and MVD, irrespective of their LA size or other comorbidities.

\section{Webcast}

You can watch a Webcast of this AATS meeting presentation by going to: http://webcast.aats.org/2016/Video/ Tuesday/05-17-16_Hall_E_1707_Labin-800.mp4.

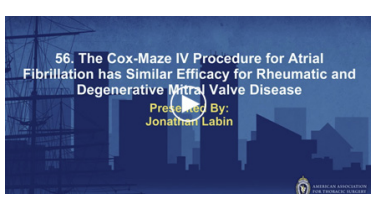

\section{Conflict of Interest Statement}

Dr Damiano receives research grants and educational funding from AtriCure and Edwards. Dr Moon receives research funding and grants from Medtronic. All other authors have nothing to disclose with regard to commercial support.

\section{References}

1. Chugh SS, Havmoeller R, Narayanan K, Singh D, Rienstra M, Benjamin EJ, et al. Worldwide epidemiology of atrial fibrillation: a Global Burden of Disease 2010 Study. Circulation. 2014;129:837.

2. Cox JL, Schuessler RB, D’Agostino HJ Jr, Stone CM, Chang BC, Cain ME, et al. The surgical treatment of atrial fibrillation. III. Development of a definitive surgical procedure. J Thorac Cardiovasc Surg. 1991;101:569-83.

3. Fujita T, Kobayashi J, Toda K, Nakajima H, Iba Y, Shimahara Y, et al. Long-term outcome of combined valve repair and maze procedure for nonrheumatic mitral regurgitation. J Thorac Cardiovasc Surg. 2010;140:1332-7.

4. Bando K, Kobayashi J, Kosakai Y, Hirata M, Sasako Y, Nakatani S, et al. Impact of Cox maze procedure on outcome in patients with atrial fibrillation and mitral valve disease. J Thorac Cardiovasc Surg. 2002;124:575-83.

5. Lawrance CP, Henn MC, Miller JR, Sinn LA, Schuessler RB, Damiano RJ Jr. Comparison of the stand-alone Cox-Maze IV procedure to the concomitant Cox-Maze IV and mitral valve procedure for atrial fibrillation. Ann Cardiothorac Surg. 2014;3:55.

6. Gammie JS, Haddad M, Milford-Beland S, Welke KF, Ferguson TB Jr, O’Brien SM, et al. Atrial fibrillation correction surgery: lessons from the Society of Thoracic Surgeons National Cardiac Database. Ann Thorac Surg. 2008;85:909-14.

7. Kim KC, Cho KR, Kim YJ, Sohn DW, Kim KB. Long-term results of the Cox-Maze III procedure for persistent atrial fibrillation associated with rheumatic mitral valve disease: 10-year experience. Eur J Cardiothorac Surg. 2007;31:261-6.

8. Lee JW, Park NH, Choo SJ, Jo MS, Song H, Song MG. Surgical outcome of the maze procedure for atrial fibrillation in mitral valve disease: rheumatic versus degenerative. Ann Thorac Surg. 2003;75:57-61.

9. Ishii Y, Nitta T. Atrial fibrillation surgery for patients with rheumatic valve disease. J Interv Card Electrophysiol. 2007;20:109-12.

10. Carapetis JR, Steer AC, Mulholland EK, Weber M. The global burden of group A streptococcal diseases. Lancet Infect Dis. 2005;5:685-94.

11. Voeller RK, Bailey MS, Zierer A, Lall SC, Sakamoto SI, Aubuchon K, et al. Isolating the entire posterior left atrium improves surgical outcomes after the Cox maze procedure. J Thorac Cardiovasc Surg. 2008;135:870-7.

12. Calkins H, Brugada J, Packer DL, Cappato R, Chen SA, Crijns HJ, et al. HRS/ EHRA/ECAS expert consensus statement on catheter and surgical ablation of atrial fibrillation: recommendations for personnel, policy, procedures and follow-up. Europace. 2007;9:335-79. 
13. Chao TF, Lin YJ, Tsao HM, Tsai CF, Lin WS, Chang SL, et al. CHADS2 and CHA2DS2-VASc scores in the prediction of clinical outcomes in patients with atrial fibrillation after catheter ablation. J Am Coll Cardiol. 2011;58:2380-5.

14. Fukada J, Morishita K, Komatsu K, Sato H, Shiiku C, Muraki S, et al. Is atrial fibrillation resulting from rheumatic mitral valve disease a proper indication for the maze procedure? Ann Thorac Surg. 1998;65:1566-9.

15. Saito T, Tamura K, Uchida D, Saito T, Togashi M, Nitta T, et al. Histopathological features of the resected left atrial appendage as predictors of recurrence after surgery for atrial fibrillation in valvular heart disease. Circ J. 2007;71:70-8.

16. Stulak JM, Sundt TM III, Dearani JA, Daly RC, Orsulak TA, Schaff HV. Ten-year experience with the Cox-Maze procedure for atrial fibrillation: how do we define success? Ann Thorac Surg. 2007;83:1319-24.

17. Gaynor SL, Schuessler RB, Bailey MS, Ishii Y, Boineau JP, Gleva MJ, et al. Surgical treatment of atrial fibrillation: predictors of late recurrence. J Thorac Cardiovasc Surg. 2005;129:104-11.

18. Gillinov AM, Sirak J, Blackstone EH, McCarthy PM, Rajeswaran J, Pettersson G, et al. The Cox maze procedure in mitral valve disease: predictors of recurrent atrial fibrillation. J Thorac Cardiovasc Surg. 2005;130:1653-60.

19. Saint LL, Bailey MS, Prasad S, Guthrie TJ, Bell J, Moon MR, et al. Cox-Maze IV results for patients with lone atrial fibrillation versus concomitant mitral disease. Ann Thorac Surg. 2012;93:789-95.

20. Weimar T, Bailey MS, Watanabe Y, Marin D, Maniar HS, Schuessler RB, et al. The Cox-maze IV procedure for lone atrial fibrillation: a single center experience in 100 consecutive patients. J Interv Card Electrophysiol. 2011;31:47-54.

21. Damiano RJ, Schwartz FH, Bailey MS, Maniar HS, Munfakh NA, Moon MR, et al. The Cox maze IV procedure: predictors of late recurrence. J Thorac Cardiovasc Surg. 2011;141:113-21.

22. Ad N, Henry L, Holmes SD, Stone LE, Hunt S. The association between early atrial arrhythmia and long-term return to sinus rhythm for patients following the Cox maze procedure for atrial fibrillation. Eur J Cardiothorac Surg. 2013;44:295-300.

23. Byrd GD, Prasad SM, Ripplinger CM, Cassilly TR, Schuessler RB, Boineau JP, et al. Importance of geometry and refractory period in sustaining atrial fibrillation: testing the critical mass hypothesis. Circulation. 2005;112:17-13.

Key Words: atrial fibrillation, arrhythmia therapy, ablation, rheumatic, mitral valve disease

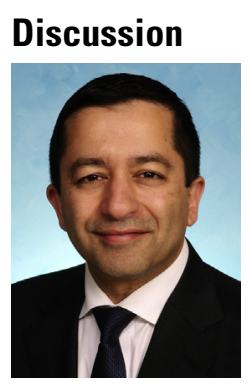

Dr Vinay Badhwar (Morgantown, $W V a)$. First, I want to specifically congratulate medical student Jonathan Labin on his first international presentation. We are unanimous in our pride and support when medical students present at AATS. I also recognize the series of superb contributions from Washington University and the leadership of your senior author, our friend and colleague, Dr Ralph Damiano, in this field to which he has been ardently dedicated.

The central message of this paper is that at the time of mitral surgery, the results after concomitant surgical ablation of the rheumatic atrium are no different than in nonrheumatic degenerative mitral disease. This, of course, provides a substantial counterargument to the prevailing knowledge and clinical practice that has established the fact that these patients are indeed different. The authors are to be congratulated on this interesting and provocative contribution. However, I would like to provide a few considerations on the study design that may impact interpretation of the results.

This represents a sample of only 245 patients from 2001 to 2015 undergoing a concomitant mitral operation and surgical ablation, or an average of just over 1 case a month, from an institution known for the management of these cases. The repair rate was only $82 \%$ for pure degenerative disease, and $9 \%$ for rheumatic disease. It is true that the average duration of atrial fibrillation (AF) in the rheumatic group was indeed longer at nearly 4 years versus 1.5 years in the degenerative group and $80 \%$ had significant heart failure versus only $50 \%$ in the rheumatic and degenerative groups, respectively.

But to the suggestion in the manuscript that the rheumatic patients were much worse by other common known metrics for $\mathrm{AF}$ analyses, such as body mass index, left atrium diameter, age, and AF type, we may wish to take a little more careful look at the make-up of each cohort. The body mass index in the rheumatic patients was only $2 \mathrm{~kg} / \mathrm{cm}^{2}$ larger; the average left atrium diameter was only $.5 \mathrm{~cm}$ larger; the rheumatic patients were nearly half the age of the degenerative patients; and, most importantly, $40 \%$ of the rheumatic patients were paroxysmal. We should note in the interpretation of the messaging of this paper that the rheumatic patients studied in this analysis are not the patients with the end-stage, dilated, 7-cm calcified atria we more commonly seen in middle-aged or older patients in North America or those encountered in non-Western countries on an almost daily basis. This is perhaps a younger population with smaller atria, earlier in the disease course, than noted in the existing literature of surgical ablation in rheumatic mitral disease.

When we specifically look at the interpretative subtext of these patients to existing rheumatic patients in the AF literature, this is not really an apples and oranges comparison, but maybe it is a Granny Smith versus Fuji apple comparison.

The 3 areas I want to highlight in the questions pertain to anticoagulation and stroke, monitoring heterogeneity, and follow-up duration. I will ask them in order and you can respond together.

First, regarding anticoagulation management and stroke risk, we know rheumatic patients with valvular AF are often at higher thromboembolic risk, not only from the left atrial appendage, but also from the stenotic valve and atrial hypokinesia. In this study, when you combine short-term permanent stroke and long-term thromboembolic events, there were a surprising 9 out of 92 , or $9.8 \%$, in the rheumatic patients and $7.2 \%$, or 11 cases, in the degenerative group. In the methods section you note that warfarin was discontinued at 3 to 6 months if the patients were in normal sinus rhythm or if atrial emptying was documented on echo. Given the nontrivial thromboembolic complication rate, please note how atrial contraction was truly assessed, how consistently this was performed, and do you believe inconsistency of atrial contraction documentation may be linked to the interpretation of the results?

Second, regarding monitoring heterogeneity, you note that several patients received actually electrocardiogram monitoring as part of the recorded follow-up. As you know, by 
Heart Rhythm Society guidelines, 24-hour Holter monitoring or interrogation of the implantables is the current standard of care reporting rhythm endpoint. How many patients had their atrial tachyarrhythmia assessed by electrocardiogram, and if these patients were eliminated from the analysis, how would this impact your results? Furthermore, you note that $62 \%$ had atrial tachyarrhythmia events. Were these counted as early failure or was your rhythm endpoint defined only by that at last follow-up?

Third, regarding the follow-up duration, we recognize that rheumatic atria have substantially higher risk of AF and these patients may be ultrastructurally different than patients with degenerative mitral disease.

You now laudably report 5-year results, yet with a median follow-up of 3 years. We see from your presentation that in a small number of patients in the fifth year of follow-up, only $50 \%$ were in normal sinus rhythm. Please comment if you think that we should preface the interpretation of your excellent results within the cautionary context of short follow-up for this specific disease state and with the possibility that your rhythm endpoint curves may diverge towards significance over time.

I thank the Association for the privilege of discussing this paper.

Jonathan E. Labin (St Louis, Mo). Thank you, Dr Badhwar, for those provocative questions and important comments. First, to address your question regarding how atrial contraction was assessed, every patient at 2 to 3 months received a transthoracic echocardiogram, and atrial emptying was assessed by the absence of atrial stasis or smoke. Additionally, prolonged monitoring was performed at 3 months, 6 months, 12 months, and annually for life.

Regarding both the inconsistency of atrial contraction documentation and whether or not this could be linked to our high thromboembolic rate, it is true that in our study the thromboembolism rate was quite high in both cohorts. Indeed, previous series from our own institution have demonstrated a stroke rate of approximately $0.2 \%$ per year following a Cox-Maze procedure. Thus, our results are quite surprising.

However, I think that this could be due to the specific patient population that we were analyzing. The recent REMEDY study by Zühlke and colleagues reported a stroke prevalence of approximately 7\% worldwide and greater than $14 \%$ in developed countries in patients with rheumatic heart disease. Moreover, similarly focused studies, with a large proportion of patients suffering from rheumatic heart disease, have shown a comparable stroke prevalence of approximately $6 \%$ after a Cox-Maze Procedure.
Most importantly, a number of studies have shown that the only risk factor for postoperative stroke following the Cox-Maze procedure is a preoperative history of stroke. In our patient sample, this percentage was quite high compared with other studies; approximately $16.3 \%$ and $18.5 \%$ in the degenerative and rheumatic cohorts, respectively.

Concerning your question of how many patients had their rhythm endpoint assessed by electrocardiogram; of our total sample, $16 \%$ of patients received only electrocardiogram monitoring. It should be noted that the majority received prolonged follow-up. However, we have not yet assessed the rate of $\mathrm{AF}$ recurrence with these specific patients removed from the sample. This is an excellent point and a future analysis that we will definitely consider performing. It should be noted that there was a similar breakdown in monitoring between the two cohorts and therefore we did not redo the analysis.

As for the $62 \%$ of early atrial tachyarrhythmias that you mentioned: Because these events occurred within the 3month blanking period, they are not considered failures, as defined by the Heart Rhythm Society consensus statement guidelines. And while $62 \%$ is a large percentage, the vast majority of these early atrial tachyarrhythmia events occurred within the first month following surgery. Additionally, and perhaps more importantly, of those patients that experienced early atrial tachyarrhythmias after the procedure, the majority remained free from late recurrence at 4 and 5 years.

Lastly, you commented on the results tailing off at 5 years and the Fuji apples, especially concerning the generalizability of our results. Given that this is one of the few studies that have documented long-term follow up of patients with rheumatic mitral valve disease who received a Cox-Maze procedure, I think that we should proceed with cautious optimism. There is no doubt that further studies need to be conducted concerning this patient population.

However, with that said, I think our sizable sample sizeparticularly during the first few years of follow-up-and the similarities in success between the 2 cohorts should encourage the wider application of surgical ablation independent of the underlying etiology of mitral valve disease. Concerning our 5-year follow-up time point specifically, this perceived drop in success is only evident when comparing freedom from AF and antiarrhythmic drugs. At 5 years, freedom from AF on and off antiarrhythmic drugs remains $72 \%$ in both cohorts. Moreover, this difference is likely due to the small sample size at 5 years and a response bias. 